\title{
Tridimensional assessment of adductor spasmodic dysphonia pre- and post-treatment with Botulinum toxin
}

\author{
P. H. Dejonckere $\cdot$ K. J. Neumann • \\ M. B. J. Moerman · J. P. Martens • \\ A. Giordano $\cdot$ C. Manfredi
}

Received: 12 October 2011/Accepted: 12 December 2011/Published online: 31 December 2011

(C) The Author(s) 2011. This article is published with open access at Springerlink.com

\begin{abstract}
Spasmodic dysphonia voices form, in the same way as substitution voices, a particular category of dysphonia that seems not suited for a standardized basic multidimensional assessment protocol, like the one proposed by the European Laryngological Society. Thirty-three exhaustive analyses were performed on voices of 19 patients diagnosed with adductor spasmodic dysphonia (SD), before and after treatment with Botulinum toxin. The speech material consisted of 40 short sentences phonetically selected for constant voicing. Seven perceptual parameters (traditional and dedicated) were blindly rated by a panel of experienced
\end{abstract}

P. H. Dejonckere ( $\square)$

University Medical Centre, ORL Phoniatrics,

Utrecht University, Utrecht, The Netherlands

e-mail: ph.dejonckere@umcutrecht.nl

P. H. Dejonckere

Federal Institute of Occupational Diseases, Brussels, Belgium

P. H. Dejonckere

Catholic University of Leuven, Neurosciences, Leuven, Belgium

K. J. Neumann

Department of Phoniatrics and Pediatric Audiology,

ENT-Clinic, University of Bochum, Bochum, Germany

M. B. J. Moerman

J. Palfijn Hospital, Ghent, Belgium

M. B. J. Moerman

Utrecht University, Utrecht, The Netherlands

J. P. Martens

University of Ghent, Ghent, Belgium

A. Giordano - C. Manfredi

Department of Electronics and Telecommunications,

University of Florence, Florence, Italy clinicians. Nine acoustic measures (mainly based on voicing evidence and periodicity) were achieved by a special analysis program suited for strongly irregular signals and validated with synthesized deviant voices. Patients also filled in a VHI-questionnaire. Significant improvement is shown by all three approaches. The traditional GRB perceptual parameters appear to be adequate for these patients. Conversely, the special acoustic analysis program is successful in objectivating the improved regularity of vocal fold vibration: the basic jitter remains the most valuable parameter, when reliably quantified. The VHI is well suited for the voice-related quality of life. Nevertheless, when considering pre-therapy and post-therapy changes, the current study illustrates a complete lack of correlation between the perceptual, acoustic, and self-assessment dimensions. Assessment of $\mathrm{SD}$-voices needs to be tridimensional.

Keywords Spasmodic dysphonia $\cdot$ Assessment . Perceptual evaluation · VHI · Voice acoustics · Botulinum

\section{Introduction}

'Adductor spasmodic dysphonia' (SD) is a focal laryngeal dystonia mainly resulting in a strained-strangled and harsh voice quality with spasms and effortful speech production [1]. $\mathrm{SD}$-voices form, in the same way as substitution voices, a particular category of voices that seems not suited for a standardized basic multidimensional assessment protocol, like the one proposed by the European Laryngological Society [2]. Traditional means of voice assessment do not adequately measure either the disease severity or the treatment outcomes [3]. SD-voices request specific parameters, particularly acoustic ones [2, 4], as their deviant acoustic characteristics [5] cannot be adequately analyzed by 
traditional software programs, particularly in running speech. Furthermore, these patients are known to report high impacts of their voice problem on their quality of life [6]. The present study deals with a tridimensional assessment approach of SD: perceptual rating with traditional and dedicated parameters, objective acoustic assessment using a specific program, and self-reported voice-related quality of life. The purpose is to check and select the most appropriate parameters for SDvoices, particularly their specificity with respect to the basic protocol for common dysphonias. The videostroboscopic and aerodynamic dimensions were left out for obvious reasons: in case of SD, laryngeal morphology is normal, and the vocal fold vibration frequently too unstable for quantifying reliably parameters as closure, regularity, and mucosal wave. Similarly, the phonation quotient (vital capacity/maximal duration of a sustained vowel) is unreliable due to major variations from trial to trial. Measurement of phonatory flow is theoretically possible with ad hoc instrumentation, but is not suited for current clinical practice, and there is no reported experience in the literature.

The standard treatment for the patients in this study was a bilateral injection of 5 International Units Botulinum (Botox ${ }^{\circledR}$, Allergan) in the vocalis muscles, via the cricothyroid membrane and under fiberendoscopic control.

\section{Materials and methods}

Thirty-three analyses were performed: 24 voice samples are originating from 12 patients diagnosed with spasmodic dysphonia (SD) by (at least) two experienced laryngologists, and analyzed (just) pre- and (a few weeks) posttreatment. Seven patients had no post-treatment analysis. Two patients had two pre-recordings at different moments, with a time interval of several months. There were 11 females and 8 males. Mean age was $60.6( \pm 9.3)$.

All patients read a standardized list of 40 short German sentences, phonetically selected for being constantly voiced. This is supposed to increase the sensitivity for detecting interruptions of vocal fold vibrations induced by the SD. Duration of reading is about $2^{\prime} 30^{\prime \prime}$.

The digital recording was made with a sampling frequency of $44.100 \mathrm{~Hz}$, in a quiet room.

\section{Perceptual parameters}

They are scored on a scale $0-10,0$ meaning the worst possible rating, and 10 the best possible one. Scoring was performed blindly and independently by three experienced voice clinicians, and scores were averaged. When for a given patient two recordings were available, ratings were made comparatively, but without knowledge of the condition (pre- or post-).
Traditional perceptual voice characteristics [2] :

$\mathrm{G}$ (Grade): overall impression of quality, integrating all specific characteristics.

B (Breathiness): audible unintended additive turbulent noise.

$\mathrm{R}$ (Roughness): the impression of irregular Fo, creakiness, harshness, including perception of individual acoustic impulses (fry).

\section{Perceptual voice characteristics dedicated to SD:}

I (Intelligibility): actually the impression of intelligibility: to what extent can the message be correctly understood?

F (Fluency): smoothness of speech production.

Vo (Voicing): in the sense that the speech is voiced or unvoiced when it actually needs to be voiced or unvoiced.

$S$ (Spasmodicity): it means the specific perceptual characteristic of adductor spasmodic dysphonia, combining strain, perception of spasms and tremor.

$\mathrm{I}, \mathrm{F}$, and Vo are taken over from the INFVo rating scale developed for and investigated on substitution voices [7] and already tried out on SD-voices [8].

\section{Acoustic parameters}

An analysis program "AMPEX" (Auditory Model Based Pitch Extractor) created by Van Immerseel and Martens [9] (and further developed until very recently) was used for the acoustic measurements. It has proven to be able to extract in a valid way the period in irregular signals with background noise. It also detects low frequency components $(<0.1 \mathrm{kHz})$, is suited for running speech and has been efficiently used for substitution voices [10]. A characteristic of this program is that it includes the three deviant acoustic events that were found relevant for characterizing SD: aperiodicity, phonatory breaks, and frequency shifts, without requesting subjective intervention of an experimenter for placing cursors and identifying deviant events, as in the experiment of Sapienza et al. [5].

The acoustic analysis is performed in three stages. In the first stage, short-term acoustic features are extracted every $10 \mathrm{~ms}$ by the auditory model described in [9]. Then these features are employed to distinguish speech frames from background (silence) frames. Finally, a global analysis of the short-term acoustic feature patterns over the entire recording is performed to produce a limited set of features that is expected to characterize the voice of the recorded speaker.

Every $10 \mathrm{~ms}$, the auditory model produces a set of more than 30 features, but for the present study, only 4 of them are relevant, namely, the energy $(\mathrm{E})$, the voicing evidence 
(VE), the voiced/unvoiced nature (VU), and the pitch frequency ( $\mathrm{Fo}$ ) (in case of voicing) of the frame. The reader is referred to [5] for more details as to how these features are actually computed.

The speech/background classification of the frames is based on an analysis of the smoothed energy pattern. The smoothed energy of frame $i$ is computed as the mean of the energies in frames $i-2$ to $i+2$. In a first step, a background threshold is determined as 1.1 times the minimal energy plus 0.05 times the maximum energy found in the recording. All frames exceeding this threshold are initially labeled as speech and the others as background. However, to avoid that too many weak parts of speech (e.g., closures of plosives, weak consonants) are classified as background, any interval shorter than $100 \mathrm{~ms}$ that was labeled as background is converted to speech again.

The first feature emerging from the global analysis stage characterizes the ability of the speaker to produce voicing. It comes in two flavors: the proportion of voiced frames (PVF) in the entire recording and the proportion of voiced speech frames (PVS). Because pauses and weak speech sounds are typically unvoiced, PVS is expected to be larger than PVF.

The second feature is the average voicing evidence (AVE) in the voiced frames. It characterizes the degree of regularity/ periodicity in the voiced frames. Since the real background frames are normally unvoiced, the analysis is performed on all frames, and not just on the speech frames, in the hope to be more robust against possible errors of the speech/background classification, which is after all purely energy based, whereas the voicing evidence is derived from an analysis of all the subband signals created by the auditory model.

The third feature is the traditional 'Jitter': JIT and JITc (corrected jitter) represent the Fo-jitter in all voiced frame pairs (=two consecutive frames) and in the voiced frame pairs with a reliable Fo in each of the two frames. The formula, which is used to compute the jitter, is:

$$
\begin{aligned}
& \text { Jitter }=\text { sum of } \operatorname{VE}(i) \times|\mathrm{T} 0(i)-\mathrm{T} 0(i-1)| / \text { sum of } \operatorname{VE}(i) \\
& \quad \times \mathrm{T} 0(i-1), \quad \mathrm{T} 0=1 / \mathrm{F} 0
\end{aligned}
$$

A fourth feature is the 90th percentile (VL 90) of the voicing length distribution. It is considered a robust estimate of the maximum voicing duration. The voicing length is defined as the number of consecutive voiced frames in the data.

\section{Acoustic measurements}

With AMPEX, the following features have been estimated:

PVF/PVS: PVF is the proportion of voiced frames and depends on the pauses appearing in speech. In addition, the PVS, the proportion of voiced speech frames is computed, thus considering only frames that are classified as speech in the first step of the analysis. Since pauses and weak sounds are typically unvoiced, PVS will typically be larger than PVF. For sustained vowels it should be expected that PVS $=\mathrm{PVF}=100 \%$ in a normal voice. For constantly voiced sentences, the rule is: the better the voice, the highest the percentages. AVE: the average voicing evidence in voiced frames. The more regular (periodic) the voiced frames, the higher the AVE.

VL 90: the 90th percentile of the voicing length distribution. The voicing length is defined as the number of consecutive voiced frames found in the data. The 90th percentile of the voicing length distribution may be considered a robust estimate of the maximum voicing duration. Phonatory breaks decrease the value of this feature.

JIT and JITc: the cycle-to-cycle period perturbation and the corrected cycle-to-cycle period perturbation. JIT represents the Fo-jitter in all voiced frame pairs $(=2$ consecutive frames), and JITc the Fo-jitter in the voiced pairs with a reliable Fo in each of the two frames.

JITN and JITNc: there is also a jitter feature which is computed without applying the VE (voicing evidence)weighting.

PVFU: the percentage of frames with an "unreliable" Fo. For example, observed sudden frequency shifts suggest that the Fo estimate is unreliable.

Further, the total duration required for reading the 40 sentences was also measured in seconds.

\section{Self-evaluation parameters}

The voice handicap index (VHI), developed by Jacobson et al. [11] is a widespread instrument for measuring the psychosocial handicapping effect of a voice disorder over three domains, the Physical (P), the Emotional (E), and the Functional $(\mathrm{F})$ domain. It is a disease-specific quality of life instrument and consists of 30 items/statements (10 in each domain), which are to be scored from 0 to 4 with a maximum score of 120 . The higher the score, the more there is a self-perceived handicapping effect caused by the voice disorder.

\section{Results}

Pre-post comparisons

\section{Perceptual parameters}

Average correlation coefficients among raters are between $0.72(\mathrm{~F})$ and $0.85(\mathrm{G})$. 
Distributions of perceptual ratings may be considered as Gaussian. A first ANOVA with pre-/post- as grouping variable shows a significant difference between the two situations for traditional $(p=0.0074)$ as well as for dedicated $(p=0.017)$ perceptual parameters, when considered globally. The effect is mainly due to $G$ and S. Figure 1 reflects the pre-post changes in perceptual parameters. Actually, in average, there is a 'post-' improvement for each parameter considered individually.

Paired comparisons (Wilcoxon test) reveal a significant post- versus pre-improvement of voice quality with the combined traditional perceptual ratings $(p=0.003)$ and with the combined dedicated perceptual ratings $(p=0.01)$.

\section{Acoustic parameters}

For acoustic parameters, the observed distribution may not always be considered as normal. Further, as acoustic parameters have different units, a z-transformation was first achieved (and, when relevant, a sign inversion) before creating two combined acoustic scores: one pertaining to the "voicing" concept (PVF, PVS, AVE, and VL90) and one pertaining to the "irregularity" concept (JIT, JITc, JITN, JITNc, and PVFU). Figure 2 shows the individual box plots for the cluster of voicing parameters. For the combined score of 'voicing' parameters, no significant difference appears between pre- and post analyses. In addition, when a paired comparison is achieved (Fig. 3), five subjects obtain a lower voicing score 'post', while seven subjects obtain a higher score (no significance). In contrast, Fig. 4 indicates that globally all parameters related to aperiodicity reveal lower values after treatment. The combined aperiodicity score 'post' is significantly lower than 'pre' (Wilcoxon test for paired values: $p=0.01$ ) (Fig. 5).

The total duration required for reading the 40 sentences was in average $154.17( \pm 26.3)$ s pre-treatment and 148.5 $( \pm 24.7)$ s post-treatment. This shortening is however not significant.

\section{Self-evaluation}

Figure 6 shows a histogram of the pre- and post-treatment VHI-scores: the observed averaged reduction in VHI-score is 15.42 points, from $64.17( \pm 21.98)$ to $48.75( \pm 22.54)$.

A paired pre-/post comparison also demonstrates a significant improvement in voice-related quality of life $(p=0.039)$, although three patients report a higher VHIscore post-treatment. Figure 7 shows the individual effects.

Correlations between changes

\section{Correlation matrix}

Table 1 shows the correlation matrix of post-pre differences for traditional and dedicated perceptual parameters, combined aperiodicity scores, and VHI-scores: no significant correlation at all is observed.

\section{Discussion}

There is no 'gold standard' for assessing severity of adductor SD. However, treatment with Botulinum injection is universally considered as the 'gold standard' symptomatic treatment for adductor SD [1, 12]. This means that a priori the perceptual evaluation parameters that show the
Fig. 1 Box plots of pre- and post-treatment ratings for the 3 traditional and the 4 dedicated perceptual parameters

\section{Perceptual parameters}

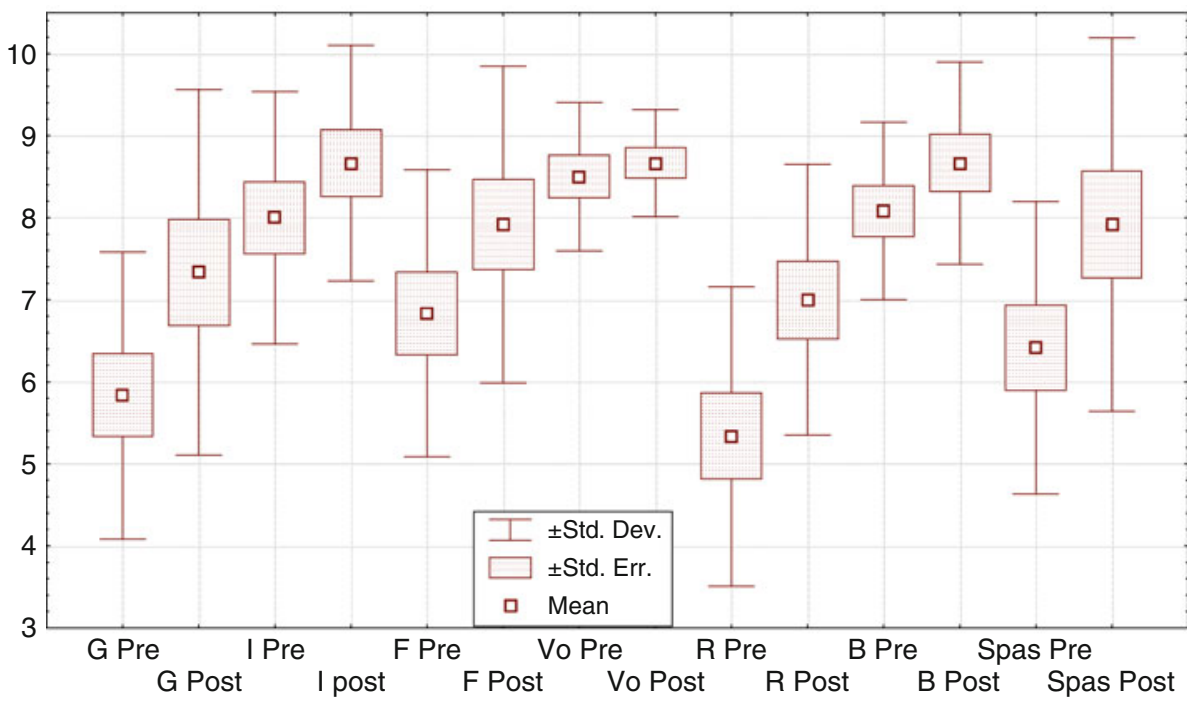


Fig. 2 Box plots of pre- and post-treatment acoustic parameters related to voicing

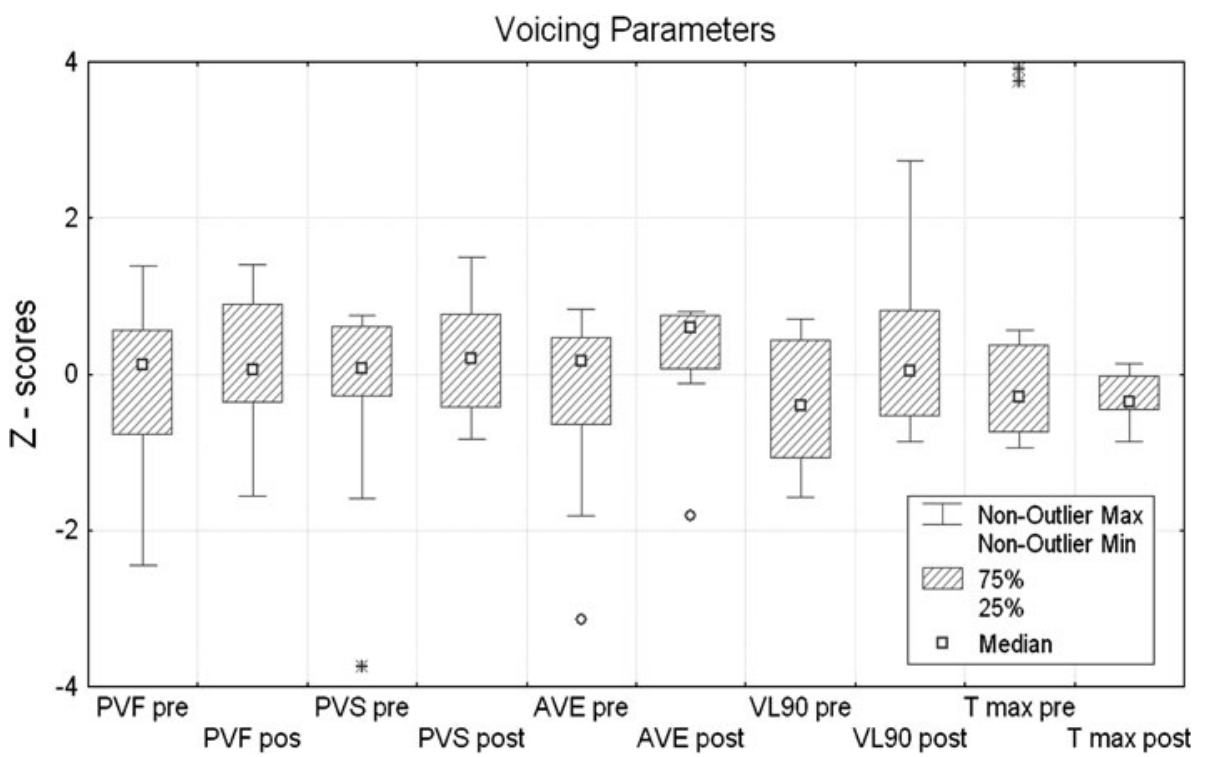

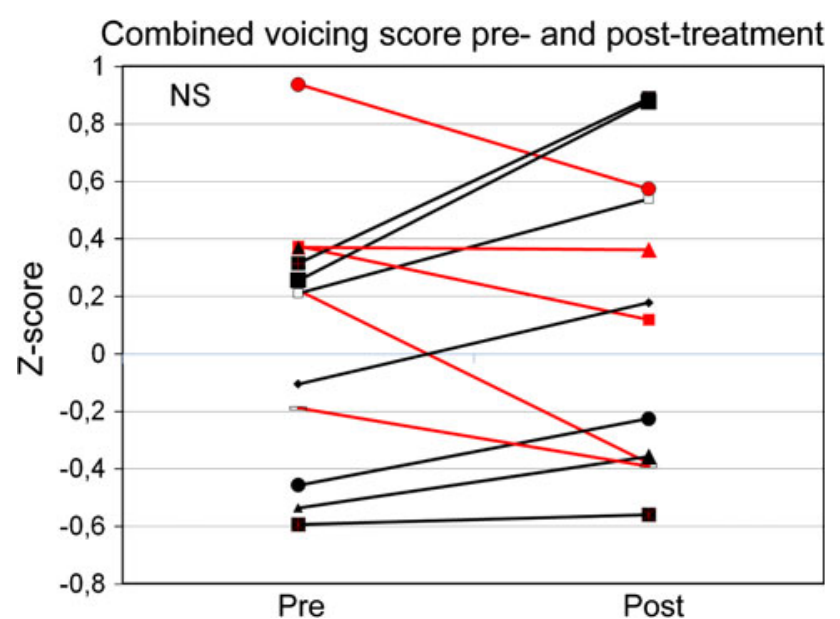

Fig. 3 Individual pre-/post-treatment changes for the combined voicing score

clearest post-/pre- changes towards a closer to normal voice may be considered as the most useful ones (face validity). The same holds for self-reported voice-related quality of life. Concerning acoustic parameters, the criteria for improvement can rely upon a physiological background: as the sentences have been phonetically selected for constant voicing, the largest post-/pre- improvement in 'voicing' indicates the voice that comes the closest to normality. The same is true for a reduction in aperiodicity: the direction to a better situation is clear (nomological validity) and makes ranking possible. The condition-particularly for aperiodicity-is that the analysis program provides valid results within the relevant range (2-6\% jitter), and this has been confirmed by a study using synthesized deviant voice signals with perfect control of the jitter 'put in' [13]. AMPEX is further not the only adequate program [13].
Perceptual parameters

In inferential statistics, an effect size helps to determine whether a statistically significant difference is a difference of practical concern. It measures the magnitude of a treatment effect. Unlike significance tests, indices for effect size are independent of sample size. In the current study, Cohen's $d$ is used [14]. Interpretation of Cohen's $d$ values is as follows [14]: a $d=0.2$ may be considered as a small effect size, a $d=0.5$, as a medium effect size, and a $d=0.8$ and more as a large effect size. This index can also be interpreted in terms of the percentage of non-overlap of the 'post'-situation scores with those of the 'pre'-situation: a $d=0.6$ corresponds to $38.2 \%$ non-overlap of pre-post scores, and a $d=1-55.4 \%$ non-overlap. This index can be used for ranking different parameters according to their discriminating power between pre- and post-treatment situations.

Table 2 shows the ranked Cohen's $d$ values for each individual perceptual parameter, as well as for the combined score for, respectively, the traditional and the dedicated parameters. The traditional parameters clearly outperform the dedicated ones, although 'spasmodicity' and 'fluency' could have some interest. However, they have not been actually validated so far. For the combined 'traditional' perceptual parameters, the Cohen's $d$ is 1.02 , corresponding to an effect size that may be considered as 'large'. For the combined 'dedicated' perceptual parameters, the Cohen's $d$ is 0.636 , corresponding to an effect size that may be considered as 'medium'.

Summarizing, it seems reasonable to keep $G$ (which includes spasmodicity and fluency), $\mathrm{R}$, and $\mathrm{B}$, as for 'common' dysphonias. B does not seem so interesting in the current study (as the evaluations are achieved after the 
Fig. 4 Box plots of pre- and post-treatment acoustic parameters related to aperiodicity

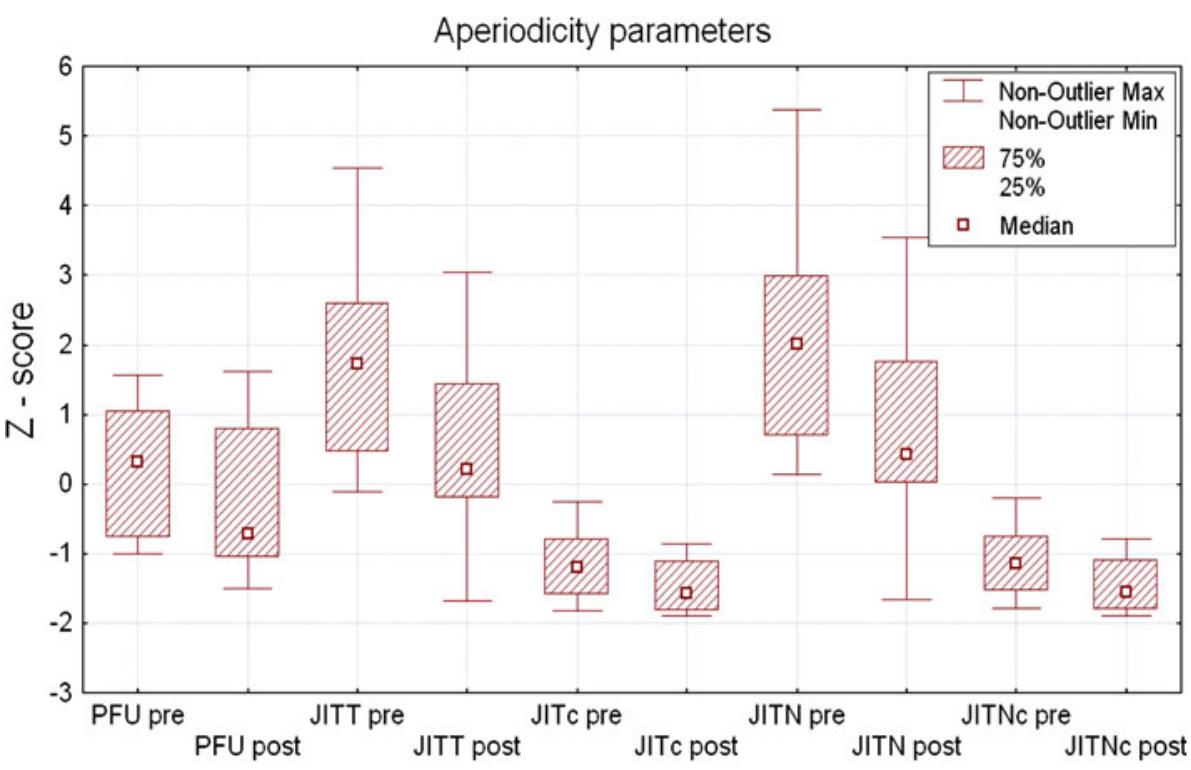

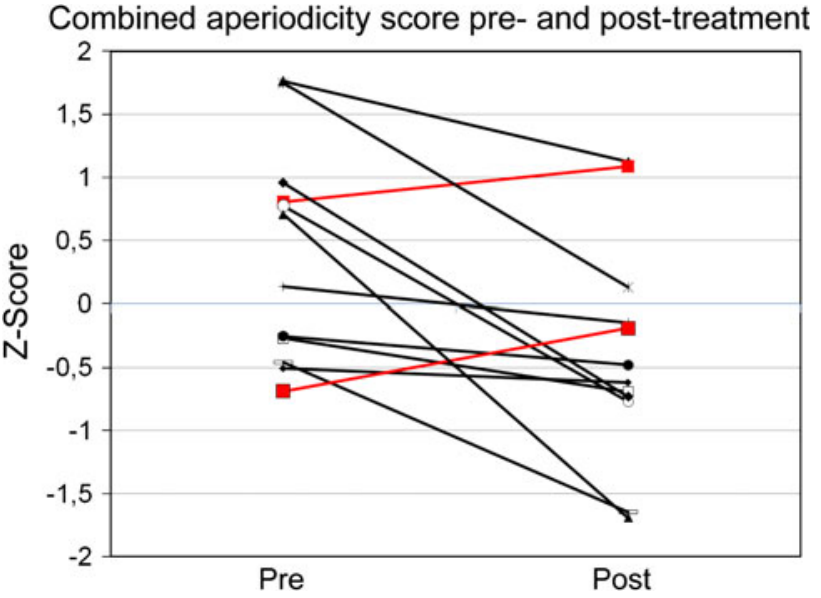

Fig. 5 Individual pre-/post-treatment changes for the combined aperiodicity score

initial post-injection phase) but has its importance in accounting for the effect of the iatrogenic paresis shortly after the Botulinum injection.

There are few quantitative reports in the literature of perceptual evaluation in Botulinum-treated SD-patients: Cannito et al. [15] found that ratings of overall perceived voice quality changed from $36 \%$ to a post-Botulinum treatment rating of $56 \%$ ( $100 \%$ being normal). In our series, the change is from 58 to $73 \%$. Cannito et al. [16] notice also that, even after treatment, voice quality and fluency remained significantly poorer in SD-patients than in healthy speakers, as is the case in our series.

Acoustic parameters

Table 3 similarly shows the ranking of the acoustic parameters, according to their $d$ values. For the combined aperiodicity score, the Cohen's $d=0.907$, corresponding to an effect size that may be considered as 'large'.

The combined voicing score has a very low value, lower than its individual components, suggesting a lack of consistency among voicing parameters.

Aperiodicity parameters are obviously best suited for acoustical assessment of SD-voices, under the mentioned condition of reliable period identification, which can currently be controlled [13]. The basic jitter algorithm appears to be adequate and sufficient. As voicing has shown to be not relevant in this context, it seems logic that weighting for voicing does not increase the discriminating performance of jitter.

Sapienza et al. [5] observed that SD-patients produced more aberrant acoustic events than controls, and that aperiodicity was the predominant acoustic event occurring during reading. Botulinum injection reduced these aperiodicities.

Self-evaluation of voice-related quality of life

The median value for the VHI-score in the general population is 6 with an asymmetrical distribution (p25 = 2; p75 $=12 ; \mathrm{p} 90=23 ; \mathrm{p} 95=32.8$ ) [17].

Regarding clinical relevant difference scores, van Gogh et al. [18] found a difference score of 10 points to be useful for individuals in clinical practice and 15 points to be useful in study group designs. In the current study, the average VHI total score is reduced by 15.42 points.

The same authors found no association between the VHI scores and the gender either for the normal population or for the voice-impaired patients. In addition, regarding age, no clear associations were present either in the normal population or in the voice-impaired patients. 
Fig. 6 Histograms of pre- and post-treatment VHI-scores with Laplace fitting curves

\section{VHI Pre-/Post Histogram \\ Laplace fitting curves}

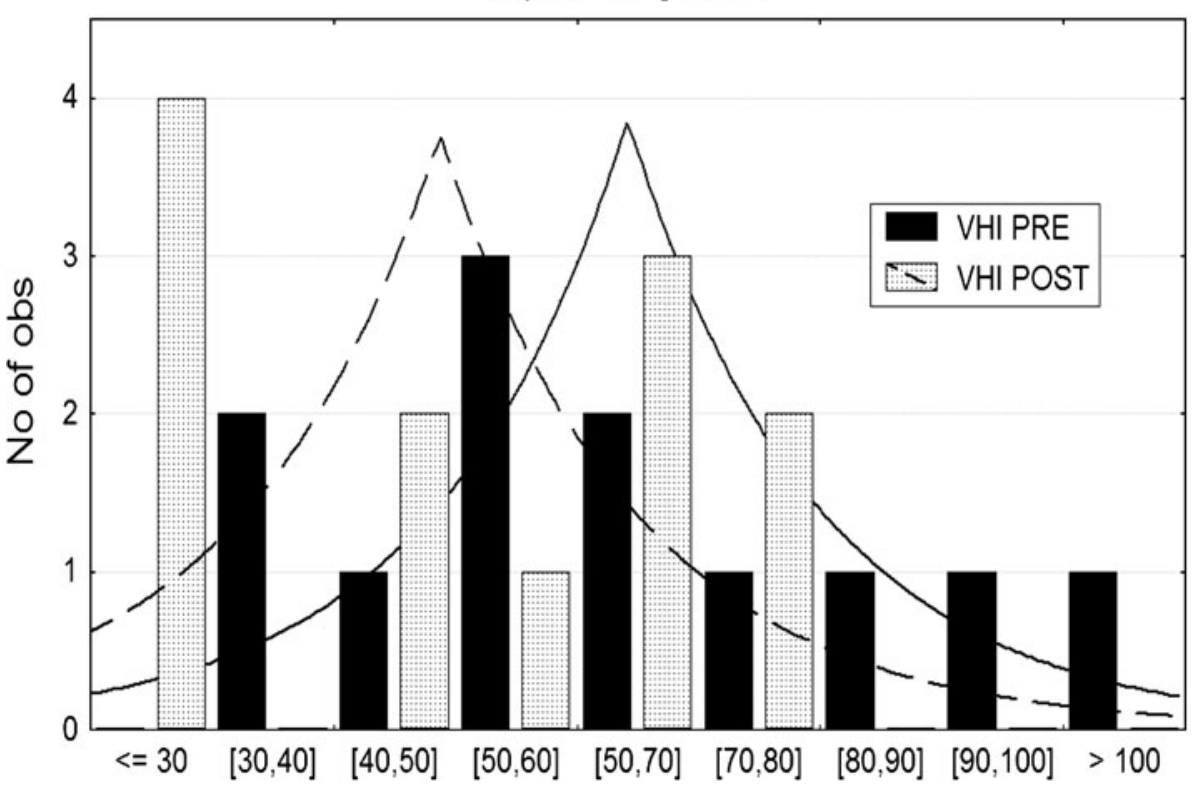

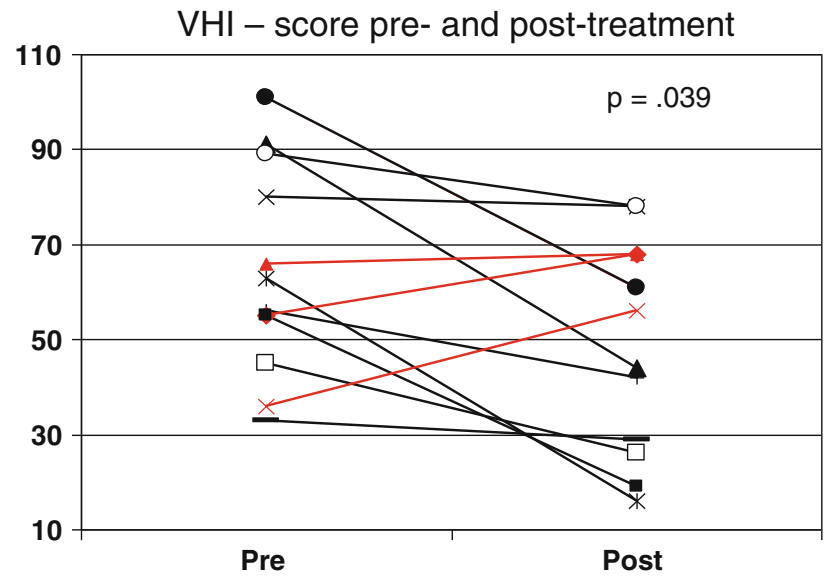

Fig. 7 Individual pre-/post-treatment changes for the VHI-score

For the VHI-score, the Cohen's $d$ is 0.692 and may be considered as medium to large.

Benninger et al. (2001) [19] found an average pretreatment score of 67.6 (64.17 in the present study), and a significant improvement after Botulinum treatment. Novakovic et al. [12] report a mean VHI improvement of 9.6\% after Botulinum toxin injection in SD-patients. In the present study, the mean improvement is $24 \%$.

\section{Correlations}

The lack of any correlation with either subjective auditory ratings or acoustic measures points out that the patient's
Table 1 Correlation matrix for pre-/post-treatment differences in perceptual, acoustic, and self-evaluation scores

\begin{tabular}{|c|c|c|c|c|}
\hline $\begin{array}{l}\text { Correlation } \\
\text { coefficient }\end{array}$ & $\begin{array}{l}\text { Difference } \\
\text { post-pre } \\
\text { traditional } \\
\text { perceptual } \\
\text { parameters }\end{array}$ & $\begin{array}{l}\text { Difference } \\
\text { post-pre } \\
\text { dedicated } \\
\text { perceptual } \\
\text { parameters }\end{array}$ & $\begin{array}{l}\text { Difference } \\
\text { post-pre } \\
\text { combined } \\
\text { aperiodicity } \\
\text { score }\end{array}$ & $\begin{array}{l}\text { Difference } \\
\text { post-pre } \\
\text { VHI- } \\
\text { score }\end{array}$ \\
\hline $\begin{array}{l}\text { Difference } \\
\text { post-pre } \\
\text { traditional } \\
\text { perceptual } \\
\text { parameters }\end{array}$ & 1 & 0.23 & -0.06 & 0.28 \\
\hline $\begin{array}{l}\text { Difference } \\
\text { post-pre } \\
\text { dedicated } \\
\text { perceptual } \\
\text { parameters }\end{array}$ & 0.23 & 1 & -0.10 & 0.39 \\
\hline $\begin{array}{l}\text { Difference } \\
\text { post-pre } \\
\text { combined } \\
\text { aperiodicity } \\
\text { score }\end{array}$ & -0.06 & -0.10 & 1 & 0.15 \\
\hline $\begin{array}{c}\text { Difference } \\
\text { post-pre } \\
\text { VHI-score }\end{array}$ & 0.28 & 0.39 & 0.15 & 1 \\
\hline
\end{tabular}

self-evaluation is a dimension that differs from the perceptual and the physical ones. This is not surprising, and is well known in the field of voice assessment in general [20]. Confronting and discussing-in an individual patientoutcomes of the different dimensions are clinically meaningful for understanding the interaction between physical, physiological, and communicative aspects of voice. 
Table 2 Ranking of effect sizes for perceptual parameters according to Cohen's $d$

\begin{tabular}{ll}
\hline Perceptual parameter & Cohen's $d$ \\
\hline $\mathrm{R}$ & 1 \\
$\mathrm{G}$ & 0.76 \\
$\mathrm{~B}$ & 0.52 \\
Combined traditional parameters & 1.03 \\
Spasmodicity & 0.73 \\
Fluency & 0.61 \\
Intelligibility & 0.41 \\
Voicing & 0.25 \\
Combined dedicated parameters & 0.65
\end{tabular}

Table 3 Ranking of effect sizes for acoustic parameters according to Cohen's $d$

\begin{tabular}{ll}
\hline Acoustic parameter & Cohen's $d$ \\
\hline JIT & 0.90 \\
JIT N & 0.78 \\
JIT N corrected & 0.78 \\
JIT corrected & 0.73 \\
PVFU & 0.52 \\
Combined aperiodicity score & 0.91 \\
VL90 & 0.72 \\
AVE & 0.57 \\
PVS & 0.41 \\
PVF & 0.31 \\
Combined voicing score & 0.15
\end{tabular}

\section{Conclusion}

Although our data set of SD-voices is limited, there arewhen comparing the pre- and post-treatment conditionssignificant perceptual, acoustical, and behavioral changes in the direction of improvement, although these three categories of outcomes miss intrinsic correlation. Clear suggestions can be made for trying out a basic dedicated protocol on larger patients' samples: for the perceptual dimension, the GRB parameters-widely used for 'common' dysphonias-remain relevant in SD-voices. In such voices, the basic jitter algorithm (rather than the voicing parameters), when applied to short constantly voiced sentences, appears well suitable for comparing a single patient with himself/herself over time. The condition is to use one of the reliable analysis programs. However, there remains a need for normative values, possibly for standardized sentences in different languages. Finally, the relevance of the VHI for quantifying the voice-related quality of life and its changes over time is confirmed.
Acknowledgments This research was partially supported by a grant from the University Medical Centre, Utrecht, for international cooperation. This research has been performed within the frame of COST2103 Action "Advanced Voice Function Assessment".

Conflict of interest There are no financial interests.

Open Access This article is distributed under the terms of the Creative Commons Attribution Noncommercial License which permits any noncommercial use, distribution, and reproduction in any medium, provided the original author(s) and source are credited.

\section{References}

1. Watts C, Nye C, Whurr R (2006) Botulinum toxin for treating spasmodic dysphonia (laryngeal dystonia): a systematic Cochrane review. Clin Rehabil 20:112-122

2. Dejonckere PH, Bradley P, Clemente P, Cornut G, CrevierBuchman L, Friedrich G, Van De Heyning P, Remacle M, Woisard V (2001) A basic protocol for functional assessment of voice pathology, especially for investigating the efficacy of (phonosurgical) treatments and evaluating new assessments techniques. Guideline elaborated by the Committee on Phoniatrics of the European Laryngological Society (ELS). Eur Arch Otorhinolaryngol 258:77-82

3. Hogikyan ND, Wodchis WP, Spak C, Kileny PR (2001) Longitudinal effects of botulinum toxin injections on voice-related quality of life (V-RQOL) for patients with adductory spasmodic dysphonia. J Voice 15:575-586

4. Dejonckere PH (2007) Critères acoustiques de fluence pour l'évaluation des dysphonies spasmodiques. In: Klein-Dallant C (ed) Voix parlée et chantée. Paris, pp 63-73. ISBN 978-29528061

5. Sapienza CM, Cannito MP, Murry T, Branski R, Woodson G (2002) Acoustic variations in reading produced by speakers with spasmodic dysphonia pre-Botox injection and within early stages of post-Botox injection. J Speech Language Hear Res 45:830-843

6. Moerman M, Lieftink A, Dejonckere PH (2008) Comparing voice pathologies with the voice handicap inventory: is a weighting factor required? Sociedad Iberoamericana de Informacion Cientifica 20.03.2008 http://www.siicsalud.com/des/expertos.php/ 89282

7. Moerman MBJ, Martens JP, Van der Borgt MJ, Peleman M, Gillis M, Dejonckere PH (2006) Perceptual evaluation of substitution voices: development and evaluation of the (I)INFVo rating scale. Eur Arch Otorhinolaryngol 263(2):183-187

8. Siemons-Lühring D, Moerman M, Martens JP, Deuster D, Müller F, Dejonckere PH (2009) Spasmodic dysphonia, perceptual and acoustic analysis: presenting new diagnostic tools. Eur Arch Otorhinolaryngol 266:1915-1922

9. Van Immerseel LM, Martens JP (1992) Pitch and voiced/ unvoiced determination with an auditory model. J Acoust Soc Am 91:3511-3526

10. Moerman MBJ, Pieters G, Martens JP, Van der Borgt MJ, Dejonckere PH (2004) Objective evaluation of quality of substitution voices. Eur Arch Otorhinolaryngol 261(10):541-547

11. Jacobson BH, Johnson A, Grywalsky C, Silbergleit A, Jacobson G, Benninger MS, Newman CW (1997) The voice handicap index (VHI): development and validation. Am J Speech Language Pathol 6:66-70

12. Novakovic D, Waters HH, D'Elia JB, Blitzer A (2011) Botulinum toxin treatment of adductor spasmodic dysphonia: longitudinal functional analysis. Laryngoscope 121:606-612 
13. Manfredi C, Giordano A, Schoentgen J, Fraj S, Bocchi L, Dejonckere PH (2011) Validity of jitter measures in non quasiperiodic voices. Part II The effect of noise. Logop Phoniatr Vocol 36:78-89

14. Cohen J (1988) Statistical power analysis for the behavioral sciences, 2nd edn. Lawrence Earlbaum Associates, Hillsdale

15. Cannito MP, Woodson GE, Murry T (1999) Perceptual scaling of spasmodic dysphonia before after botulinum toxin treatment. In: Dejonckere P, Peters HFM (eds) Communication and its disorders: a science in progress. Nijmegen University Press, Amsterdam, pp 161-163

16. Cannito MP, Woodson GE, Murry T, Bender D (2004) Perceptual analyses of spasmodic dysphonia before and after treatment. Arch Otolaryngol Head Neck Surg 130:1393-1399
17. Maertens K, de Jong FICRS (2007) The voice handicap index as a tool for assessment of the biopsychosocial impact of voice problems. B-ENT 3:61-66

18. Van Gogh CDL, Mahieu H, Kwik D, Rinkel R, Langendijk J, Verdonck-de Leew I (2007) Voice in early glottic cancer compared to benign voice pathology. Eur Arch Otorhinolaryngol 264:1033-1038

19. Benninger MS, Gardner G, Grywalski C (2001) Outcomes of botulinum toxin treatment for patients with spasmodic dysphonia. Arch Otolaryngol Head Neck Surg 127:1083-1085

20. Speyer R, Wieneke GH, Dejonckere PH (2004) Documentation of progress in voice therapy: perceptual, acoustic, and laryngostroboscopic findings pretherapy and posttherapy. J Voice 18(3):325-340 